\title{
Demystifying Typicality
}

\author{
Roman Frigg and Charlotte Werndl* \\ Forthcoming in: Philosophy of Science (PSA Proceedings)
}

\begin{abstract}
A gas prepared in a non-equilibrium state will approach equilibrium and stay there. An influential contemporary approach to Statistical Mechanics explains this behaviour in terms of typicality. However, this explanation has been criticised as mysterious as long as no connection with the dynamics of the system is established. We take this criticism as our point of departure. Our central claim is that Hamiltonians of gases which are epsilon-ergodic are typical with respect to the Whitney topology. Because equilibrium states are typical, we argue that there follows the desired conclusion that typical initial conditions approach equilibrium and stay there.
\end{abstract}

*Authors are listed alphabetically; the work is fully collaborative. An earlier version of this paper has been presented at the 2010 PSA meeting in Montreal and we would like to thank the audience for a valuable discussion. 


\section{Contents}

1 Introduction 3

2 Boltzmannian Statistical Mechanics 4

3 The Typicality Argument 5

4 Epsilon-Ergodicity Is Sufficient for Thermodynamic-like Behaviour

$\begin{array}{lll}5 & \text { The Whitney Topology } & 9\end{array}$

6 The Lennard-Jones Potential 10

$\begin{array}{lll}7 & \text { Completing the Argument } & 14\end{array}$

\begin{tabular}{ll}
\hline References & 15
\end{tabular} 


\section{Introduction}

A system prepared in a non-equilibrium state and then isolated from its environment will undergo a state transition approaching, and eventually reaching, equilibrium, the final state in which the system will stay. Well-known examples of this process include the spreading of gases, the cooling of coffee, and the uniform spreading of milk in tea. It is the aim of Statistical Mechanics (SM) to explain this aspect of the behaviour of a system in terms of the mechanical laws governing the dynamics of the system's micro constituents (i.e. the atoms or molecules from which it is made up).

An influential contemporary approach to SM explains this behaviour in terms of typicality (see, for instance, Goldstein 2001). Intuitively speaking, something is typical if it happens in the vast majority of cases: typical lottery tickets are blanks, and in typical long series of dice throws the side with four spots faces upwards with a relative frequency of $1 / 6$. The leading idea of a typicality-based version of SM is to explain why systems approach equilibrium and then stay there by showing that such behaviour is typical pretty much in the same way in which blanks and sequences with a relative frequency of $1 / 6$ are typical. However, this explanation has been criticised as mysterious as long as no connection with the dynamics of the system is established (Frigg 2009, 2010b). We take this criticism as our point of departure and make a first step towards demystifying typicality by establishing a connection with dynamics.

SM comes in different non-equivalent formulations. In what follows we restrict attention to Boltzmannian SM (BSM), which is the formalism in which typicality-based versions of SM are usually presented ${ }^{1}$ A further restriction concerns the kinds of systems we investigate. While BSM in principle covers a variety of systems, we restrict our attention to gases. The successful applications of BSM are in the theory of gases and there remain important open questions about whether, and if so how, it can be applied to liquids and solids. For this reason gases provide a good starting point.

We begin by introducing the essentials of BSM (Section 2), which paves the ground for introducing the Typicality Argument (Section 3). We then argue that epsilon-ergodicity is the sought-after dynamical property (Section 4 ) and introduce the Whitney topology as a physically relevant topology with

\footnotetext{
${ }^{1}$ For a survey of the different approaches to SM and detailed discussion of BSM see Frigg (2008).
} 
respect to which typicality claims as regards Hamiltonians should be formulated (Section 5). The central claim of the paper is that Hamiltonians which are epsilon-ergodic for the energy values relevant to gases are are typical in the class of perturbed Lennard-Jones Hamiltonians (Section 6), which puts the Typicality Argument on solid footing (Section 7).

\section{Boltzmannian Statistical Mechanics}

Consider a gas consisting of $n$ particles moving in three dimensional space. From the micro perspective the gas is a collection of molecules or atoms and its mechanical state is described by a point $x$, its microstate, in a $6 n$ dimensional phase space $\Gamma$. We assume that all particles obey the laws of classical Hamiltonian mechanics. Since by assumption the gas is isolated from its environment, its energy $E$ is conserved and the motion of the system's microstate $x$ is confined to a $6 n-1$ dimensional energy hypersurface $\Gamma_{E}$. The phase flow $\phi_{t}\left(t\right.$ denotes time) on the energy hypersurface $\Gamma_{E}$ satisfies the equations of motion, and $s_{x}: \mathbb{R} \rightarrow \Gamma_{E}, s_{x}(t)=\phi_{t}(x)$ is the solution originating in $x$. The phase space comes endowed with the Lebesgue measure $\mu$, which can be restricted to $\Gamma_{E}$. Liouville's theorem states that $\mu$ itself is preserved under the dynamics of the system, and it can be shown that the restriction of $\mu$ to $\Gamma_{E}, \mu_{E}$, is also preserved. In systems with finite energy (such a gases confined to containers) $\mu_{E}\left(\Gamma_{E}\right)$ is finite. Thus we can always normalise the measure on $\Gamma_{E}$ so that $\mu_{E}\left(\Gamma_{E}\right)=1$. In what follows we assume that $\mu_{E}\left(\Gamma_{E}\right)$ has been normalised, which has the effect that $\mu_{E}$ is a probability measure on $\Gamma_{E}$. The triple $\left(\Gamma_{E}, \mu_{E}, \phi_{t}\right)$ is a measure-preserving dynamical system.

From a macro perspective the condition of a gas is characterised by its macrostate. In keeping with a long-standing tradition, we assume that there is only a finite number of such macrostates: $M_{i}, i=1, \ldots, m$. Two of these are of particular importance: the state at the beginning of a process, the past state of the system, and the state it reaches at the end, the equilibrium state. Without loss of generality we assume that the labelling of the macrostates is such that $M_{1}$ is the past state and $M_{m}$ the equilibrium state, and to make

notion more intuitive we set: $M_{1}=M_{p}$ and $M_{m}=M_{e q}$. At the heart of BSM lies the posit that macrostates supervene on microstates (i.e. every change of the macrostate of a system must be accompanied by a change of its microstate). This is compatible with there being many microstates 
corresponding to the same macrostate. We therefore introduce the notion of a macro region $\Gamma_{M_{i}}(1 \leq i \leq m)$, which, by definition, contains all $x \in$ $\Gamma_{E}$ for which the system is in $M_{i}$. The $\Gamma_{M_{i}}$ form a partition of $\Gamma_{E}$ (i.e. they do not overlap and jointly cover $\Gamma_{E}$ ). It is the upshot of Boltzmann's (1877) combinatorial argument that $\Gamma_{M_{e q}}$ is vastly larger (with respect to $\left.\mu_{E}\right)$ than any other macro-region, a fact known as the dominance of the equilibrium macrostate. In fact it is so large that it takes up almost the entire energy hypersurface $2^{2}$ The Boltzmann entropy of a macrostate is defined as $S_{B}\left(M_{i}\right):=k_{B} \log \left[\mu\left(\Gamma_{M_{i}}\right)\right]$, where $k_{B}$ is the Boltzmann constant $(1 \leq i \leq m)$ (cf. Frigg and Werndl 2011b). It follows that $S_{B}\left(M_{p}\right) \ll S_{B}\left(M_{e q}\right)$. The Boltzmann entropy of a system at time $t, S_{B}(t)$, is the entropy of the system's macrostate at time $t$.

\section{The Typicality Argument}

One of the core challenges faced by BSM is to explain why systems, when left to themselves, approach equilibrium and stay there. Lebowitz (1993a, 1993b), Goldstein (2001) and Goldstein and Lebowitz (2004) proposed different answers which all rely in one way or another on the notion of typicality. The different arguments are discussed in Frigg (2009, 2010b) and there is no need to repeat the analysis given there. In what follows we focus on what emerged from that discussion as the most promising account, which we call the Typicality Argument. We provide a concise statement of the account, point out what is missing, and then offer a proposal of how to fill the gaps.

Before doing so, we need to make the somewhat vague notion of a system approaching equilibrium and staying there more precise. There is a temptation to base the discussion on a strict notion involving irreversibility and universality. However, as pointed out in Frigg and Werndl (2012), this is unrealistic and we should regard our mission as accomplished if we can show that gases exhibit what Lavis $(2005,255)$ calls thermodynamic-like behaviour (TD-like behaviour): the entropy of a system that is initially prepared in a low-entropy state increases until it comes close to its maximum value; it then stays there and only exhibits frequent small and rare large (downward) fluctuations. So the question is: why is it the case that systems, when left

\footnotetext{
${ }^{2}$ See Goldstein (Goldstein 2001, 45). We set aside the problem of degeneracy (Lavis 2005, 255-58).
} 
to themselves, behave TD-like? Goldstein offers the following answer to this question:

' $\left[\Gamma_{E}\right]$ consists almost entirely of phase points in the equilibrium macrostate $\left[\Gamma_{M_{e q}}\right]$, with ridiculously few exceptions whose totality has volume of order $10^{-10^{20}}$ relative to that of $\left[\Gamma_{E}\right]$. For a nonequilibrium phase point $[x]$ of energy $E$, the Hamiltonian dynamics governing the motion $[x(t)]$ would have to be ridiculously special to avoid reasonably quickly carrying $[x(t)]$ into $\left[\Gamma_{M_{e q}}\right]$ and keeping it there for an extremely long time - unless, of course, $[x]$ itself were ridiculously special.' (Goldstein 2001, 43-44) $)^{3}$

A reasonable reconstruction of this passage is that it is an argument involving three typicality claims:

Premise 1: The macrostate structure of the gas is such that equilibrium states are typical in $\Gamma_{E}$.

Premise 2: The Hamiltonian of the gas is typical in the class of all relevant Hamiltonians.

Conclusion: Typical initial conditions lie on solutions exhibiting TD-like behaviour.

This is the Typicality Argument. It presents us with various challenges. For one, there is the conceptual question of whether we can explain the behaviour of a particular system by appeal to what systems typically do. For another, there are concerns about the formulation of the above argument: what notions of typicality are at work in the two premises and how exactly does the conclusion follow from them? In what follows we set the conceptual worry aside and focus on the argument itself.

Premise 1 alludes to the dominance of the equilibrium state, and the typicality claim rests on a comparison of the Lebesgue measures of sets. So the notion of typicality involved here is a measure-theoretic one. Here typicality is a relational property of an element $e$ of a set $\Sigma$, which $e$ posses with respect to $\Sigma$, a property $P$ and a measure $\nu$ (the typicality measure). The intuitive idea is that $e$ is typical if and only if (iff) most members of $\Sigma$ have property $P$ and $e$ is one of them. In formal terms, let $\Pi$ be the subset of $\Sigma$ consisting of all elements that have property $P$. Then the element $e$ is $m$-typical iff $e \in \Pi$ and $\nu_{\Sigma}(\Pi):=\nu(\Pi) / \nu(\Sigma) \geq 1-\delta$, where $0 \leq \delta \ll 1$ is a

\footnotetext{
${ }^{3}$ Square brackets indicate that the original notation has been replaced by the notion used in this paper.
} 
very small real number. The 'm' in front of 'typical' indicates that this is a measure-theoretic notion of typicality. Conversely, an element $e$ is m-atypical iff it belongs to the complement of $\Pi$. This definition of typicality underlies the claim made in Premise 1 if we make the following associations: $\Gamma_{E}$ is $\Sigma$, $x$ is $e$, being an equilibrium state is $P, \Gamma_{M_{e q}}$ is $\Pi$ and $\mu_{E}$ is $\nu$. Then Premise 1 is true for gases: it the follows from the dominance of the equilibrium state that there is a small $\delta$ such that $\nu\left(\Gamma_{M_{e q}}\right) / \nu\left(\Gamma_{E}\right) \geq 1-\delta$. Hence, equilibrium states are m-typical in $\Gamma_{E}$.

Premise 2 is a restatement in the language of typicality of the claim that the Hamiltonian of the system is not 'ridiculously special'. But function spaces, unlike phase spaces, do not come equipped with normalized measures, and therefore the above notion of typicality cannot be used to make this claim precise. To get around this difficulty, we replace the comparison of measures in the above definition with the topological notion of comeagre. A set $A$ of a topological space $\Lambda$ is called meagre iff it is a countable union of nowheredense sets: ${ }^{4}$ a set is called comeagre iff its complement is meagre (cf. Oxtoby 1980). Loosely speaking, a comeagre set is the topological counterpart of a set of measure one, and a meagre set corresponds to a set of measure zero. We can then define a topological notion of typicality as follows. Typicality is a relational property of an element $e$ of a set $\Sigma$, which $e$ posses with respect to a set $\Sigma$, a property $P$ and a topology $\tau$ on $\Sigma$. The intuitive idea is again that $e$ is typical iff most members of $\Sigma$ have property $P$ and $e$ is one of them. Formally: let $\Pi$ be the subset of $\Sigma$ consisting of all elements that have property $P$. Then the element $e$ is t-typical iff $e \in \Pi$ and $\Pi$ is comeagre ${ }^{5}$ in the topological space $\Lambda=(\Sigma, \tau)$. The 't' indicates that this is a topological notion of typicality ${ }^{6}$ Conversely, an element $e$ is t-atypical iff it belongs to the complement of $\Pi$, which is meagre in $\Lambda$.

This definition of typicality can now be used to analyse Premise 2. Unfortunately, things are less straightforward than in the discussion of Premise 1. The problems we are up against become palpable when we try to bring to bear the abstract definition on specifics of the situation. While it is obvious that $e$ is a Hamiltonian, it is less clear what the relevant class $\Sigma$ is. Since we are focussing on gases, we could say that $\Sigma$ is the class $G$ of all gas Hamiltonians. However, from a mathematical point of view this is not

\footnotetext{
${ }^{4} \mathrm{~A}$ set $B$ is called nowhere dense iff there is no neighbourhood on which $B$ is dense.

${ }^{5}$ Comeagre sets are also called generic.

${ }^{6}$ For this notion of typicality to make sense, the set $\Sigma$ has to be large; in particular, $\Sigma$ cannot be itself meagre. The cases we consider satisfy this condition.
} 
helpful because we do not know how $G$ is circumscribed. We can of course list particular examples (or even families of examples) of gas Hamiltonians, but it is not clear whether these examples span $G$. A further problem concerns the choice of an appropriate topology $\tau$. Qualifying sets as meagre and comeagre presupposes a topology $\tau$ on $G$, and it is not a priori clear what topology one ought to choose. Finally, there is the problem of finding the relevant property $P$. In order to support the desired conclusion, $P$ has to be a dynamical property that guarantees TD-like behaviour. What is this property?

The aim of this paper is to argue that the relevant property $P$ is being epsilon-ergodic and that the relevant topology $\tau$ is the Whitney topology. We go on to argue that the Typicality Argument holds for an important subset of $G$, namely perturbed Lennard-Jones Hamiltonians. That is, $\Sigma$ is the class of perturbed Lennard-Jones Hamiltonians and $\Pi$ are Hamiltonians of this class which are epsilon-ergodic. We then suggest that a piecemeal approach where one proves typicality claims for perturbations of realistic potentials (such as the Lennard-Jones potential) is all we need because it is empirically more relevant and avoids unnecessary complications about how to circumscribe $G$.

\section{Epsilon-Ergodicity Is Sufficient for Thermodynamic- like Behaviour}

In this section we argue that being epsilon-ergodic is sufficient for TD-like behaviour. The time-average $T_{A}(x)$ of a solution originating in $x \in \Gamma_{E}$ relative to the measurable set $A$ is:

$$
T_{A}(x)=\lim _{t \rightarrow \infty} \frac{1}{t} \int_{0}^{t} \chi_{A}\left(\phi_{\tau}(x)\right) d \tau
$$

where $\chi_{A}(x)$ denotes the characteristic function of $A$ and the measure on the time axis is the Lebesgue measure. $\left(\Gamma_{E}, \mu_{E}, \phi_{t}\right)$ is ergodic iff for all measurable sets $A$ and for all $x \in \Gamma_{E}$ (except, perhaps, for a set $B$ with $\mu_{E}(B)=0$ ) we have $T_{A}(x)=\mu_{E}(A)$. A solution is said to be ergodic with respect to a measurable set $A$ iff the proportion of time it spends in $A$ equals the measure of $A$.

Now consider $B$ - the set of points which lie on non-ergodic solutions with respect to $M_{e q}$. It immediately follows that the property of being on an 
ergodic solution with respect to $M_{e q}$ in $\Gamma_{E}$ is m-typical: it is a consequence of the system being ergodic that $\mu_{E}(B)=0$ and thus $\mu_{E}\left(\Gamma_{E} \backslash B\right)=1$. Combining this result with Premise 1 yields that for ergodic systems initial conditions that lie on TD-like solutions are m-typical. The argument goes as follows. For an initial condition $x \in \Gamma_{M_{p}}$ on an ergodic solution the dynamics will carry it to $\Gamma_{M_{e q}}$ and then it will stay there most of the time (it will move out equilibrium only rarely because the non-equilibrium regions are small compared to $\Gamma_{M_{e q}}$ ). Consequently, the Boltzmann entropy of the system is close to its maximum most of the time. The set of initial conditions in $\Gamma_{M_{p}}$ that are on ergodic solutions with respect to $M_{e q}$ is $E S_{p}:=\Gamma_{M_{p}} \backslash B$. Since $\mu_{E}\left(B \cap \Gamma_{M_{p}}\right)=0$, trivially $\mu_{E}\left(E S_{p}\right) / \mu_{E}\left(M_{p}\right)=1.7$

The leading idea of epsilon-ergodicity is to relax the requirement that $\mu_{E}(B)=0$ and to allow for sets of initial conditions on non-ergodic solutions with respect to $M_{e q}$ that are small but need not be of measure zero: $\mu_{E}(B) \leq$ $\varepsilon$, where $\varepsilon \geq 0$ is a very small real number. For a detailed exposition of epsilon-ergodicity, we refer the reader to Frigg and Werndl (2011) and Vrans (1998). It is easy to see, however, that combined with Premise 1 epsilonergodicity implies that $\mu_{E}\left(E S_{p}\right) / \mu_{E}\left(M_{p}\right) \geq 1-\varepsilon$, which is the result we need. That is, for epsilon-ergodic systems initial conditions that lie on TDlike solutions are m-typical (we will see below how exactly this result is used to establish the conclusion of the above argument) 8

\section{The Whitney Topology}

In order to qualify classes of Hamiltonians as meagre or comeagre, we need a topology (intuitively speaking, a topology allows us to say how close Hamiltonians are to each other). In what follows we restrict attention to smooth Hamiltonians $H(p, q)=T(p, q)+V(p, q)$ with a fixed term $T(p, q)=p^{2} / 2 m$. So varying the Hamiltonian amounts to varying the potential energy $V(p, q)$

\footnotetext{
${ }^{7}$ It is possible to take the further step and interpret $\mu_{E}(.) / \mu_{E}\left(\Gamma_{M_{p}}\right)$ (defined for all measurable subsets of $\Gamma_{M_{p}}$ ) as probability. There is then the question about how these probabilities ought to be interpreted (for more on this see Frigg 2010a; Frigg and Hoefter 2010, Werndl 2009).

${ }^{8}$ Ergodicity and epsilon-ergodicity are silent about relaxation times. This is a virtue because some systems will approach equilibrium quicker and some slower (Frigg and Werndl 2011a). Needless to say, in order to be empirically adequate, the specific dynamical systems of SM have to show the correct relaxation times, and this has to be required alongside epsilon-ergodicity.
} 
(cf. Markus and Meyer 1969, 1974). For Hamiltonians of that kind it is physically natural to say that two Hamiltonians are close when the difference between the Hamiltonians themselves as well as all their derivatives is small. More precisely, consider two Hamiltonians $H_{1}=T+V_{1}$ and $H_{2}=T+V_{2}$. They are close when $\left\|V_{1}-V_{2}\right\|+\left\|V_{1}^{\prime}-V_{2}^{\prime}\right\|+\left\|V_{1}^{\prime \prime}-V_{2}^{\prime \prime}\right\|+\ldots$ is small, where $\|f\|$ is the integral of $|f|$ over $\Gamma$. This notion of closeness gives rise to the Whitney topology (with respect to the potential functions) on the class of smooth Hamiltonians with phase space $\Gamma$ and kinetic energy $T$ (cf. Hirsch 1976; Markus and Meyer 1969, 1974). This topology has a clear physical motivation: $T$ is the standard kinetic energy, and saying that two potentials are close if the difference of the potentials as well as all their derivatives is small is natural if one thinks about the Taylor expansion of a potential function.

\section{The Lennard-Jones Potential}

One possible interpretation of the typicality claim involved in Premise 2 is that Hamiltonians which are epsilon-ergodic for the energy values relevant to gases are comeagre in the entire class of gas Hamiltonians $G$. However, as we have seen above, $G$ is not clearly circumscribed, and even if it was, the problem is at a level of mathematical complexity that make general proofs look like a remote dream. Instead we will defend a more restriced claim as an interpretation of the typicality claim involved in Premise 2. Rather than considering the entire class of gas Hamiltonians, we focus on a limited yet important subclass of $G$ and show that in that subclass the desired result holds. The relevant subclass $L$ is the class of smooth Hamiltonians which are small perturbations (in the Whitney topology with respect to the potential function) of a Lennard-Jones Hamiltonian. We now face two challenges. First, we need to explain why $L$ is an important subclass of $G$. Second, we have to support the claim that Hamiltonians which are epsilon-ergodic for the energy values corresponding to gases are comeagre in $L$. We first introduce Lennard-Jones Hamiltonians and then provide arguments for the two claims.

The Lennard-Jones potential for two particles is:

$$
U(r)=4 \alpha\left(\left(\frac{\rho}{r}\right)^{12}-\left(\frac{\rho}{r}\right)^{6}\right)
$$

where $\alpha$ describes the depth of the potential well, $r$ the distance between 
the two particles, and $\rho$ the distance at which the inter-particle potential is 0 . The potential of the entire system is then obtained by summing over all two-particle-interactions.

The Lennard-Jones potential is important because there is good evidence that the interaction between many real gas molecules is accurately described by that potential at least to a good degree of approximation. Hence, whatever potentials $G$ comprises, many real gases cluster in a subclass of $G$, namely $L$, and so knowing how the members of $L$ behave tells us a lot about how real gases behave. What evidence is there to support this claim? Data about inter-particles forces suggest that for many real gas molecules the interaction is well described by the Lennard-Jones potential (McQuarrie 2000, 236-7; Reichl 1998, 502-5). Furthermore, Lennard-Jones gases have been studied numerically in some detail and the result is that they provide 'a relatively accurate description of the thermodynamic properties of many real molecules' Attard (2002, 156). Verlet (1967) extensively studied the thermodynamic properties such as the compressibility factor 9 of a Lennard-Jones model of an argon gas for various temperatures and densities. All properties agreed well with the ones of real argon, prompting him to conclude that there was a striking agreement between the results obtained in numerical studies of argon and the properties of real argon gas. Hansen and Verlet (1969) compared the phase transitions of real argon and the phase transitions predicted by a Lennard-Jones model of argon and also found good agreement between the two. Saxena (1957) and Thornton (1960) compared empirical and values and theoretical values of the viscosity ${ }^{10}$ and thermal conductivity ${ }^{11}$ of gases for several temperature values. They found that the Lennard-Jones model realistically describes the monatomic gases of krypton, argon, neon and helium and the binary mixtures xenon-krypton and xenon-argon. Finally, Zabaloy et al. (2006) compared diffusion of real gases where all particles are chemically identical with the diffusion predicted by a Lennard-Jones model of a gas for a wide range of temperatures and densities. They found that the Lennard-Jones model reproduces the behaviour of krypton, methane and carbon dioxide gases well.

We now address the second challenge and show that there is evidence that Hamiltonians which are epsilon-ergodic for the energy values relevant

\footnotetext{
${ }^{9}$ Intuitively speaking, the compressibility factor characterises the deviation of a gas from the behaviour of an ideal gas.

${ }^{10} \mathrm{Viscosity}$ is a measure of the resistance of a fluid.

${ }^{11}$ Thermal conductivity is the ability of a material to conduct heat.
} 
to gases are typical in L. More precisely, we aim to establish the following proposition: Hamiltonians for which the resulting dynamical systems $\left(\Gamma_{E}, \mu_{E}, \phi_{t}\right)$ are epsilon-ergodic (for the energy value corresponding to gases) are typical in $L$ with respect to the Whitney topology. But why study perturbations? Why not just study the Lennard-Jones potential itself? The answer is that experiments cannot establish that gas molecules interact with an exact Lennard-Jones potential. In fact their real interaction potential might well differ slightly from the original Lennard-Jones potential - hence the interest in perturbations. Then, to be able to make claims about real gases, we need that the relevant property is robust under perturbations. That is, what we need is that epsilon-ergodic Hamiltonians are typical in the set of all perturbed Lennard-Jones Hamiltonians.

The evidence in support of this claim is of two kinds. The first kind consists of studies of the unperturbed Lennard-Jones potential. Several numerical investigations of many particle systems whose parts interact with a Lennard-Jones potential found the motion to be epsilon-ergodic for the energy values relevant to gases. These studies have a bearing on the above claim because due to numerical roundoff errors during the numerical computation, the system evolves only approximately according to the Lennard-Jones potential. If small perturbations of Lennard-Jones gases were not epsilon-ergodic, then one would expect that the motion does not appear to be epsilon-ergodic in numerical simulations. However, the motion appears to be epsilon-ergodic, which supports the claim that small perturbations of Lennard-Jones gases are epsilon-ergodic.

Already in Frigg and Werndl (2011) evidence was collected that LennardJones gases are epsilon-ergodic. In addition to the evidence mention there, let us list some further numerical results which support the claim. Mountain and Thirumalai's (1989) numerical experiments on a two-dimensional Lennard-Jones system (all two-particles interactions were considered) show that the motion appears to be epsilon-ergodic for the energy values relevant to gases. Bennetin and Tenenbaum (1983) investigated a two-dimensional Lennard-Jones gas of identical particles with nearest neighbour interactions and found that the motion appears to be epsilon-ergodic. Yoshimura (1997) studied a one-dimensional chain of particles interacting through a LennardJones potential with nearest neighbour interactions. They found evidence for epsilon-ergodicity as well as on average exponential divergence of solutions for the energy values relevant to gases (a positive value of the largest 
Lyapunov exponent - see Robinson 1995, 86) ${ }^{12}$

It should be mentioned that in most of these studies evidence is found for a stochastic threshold (e.g., Bennetin and Tenenbaum 1983; Bocchieri et al. 1970; Yoshimura 1997). A stochastic threshold is a value of the energy above which the motion of the system is epsilon-ergodic; for energy values below the threshold the motion is not epsilon-ergodic because it is either completely regular or the energy hypersurface is broken up into a region of regular motion and a region ${ }^{13}$ where the motion appears to be random. ${ }^{14}$ For our purposes it is important to note that the energy values below the energy threshold are irrelevant. As already mentioned in Frigg and Werndl (2011a), many believe that for very low energy values the classical mechanical description will no longer adequately describe the relevant physical systems because quantum effects can no longer be ignored. Even in cases where the quantum effects can be ignored, these low energy values do not correspond to gases but to solids (e.g., Stoddard and Ford 1973).15

The second kind of evidence supporting our claim consists of studies investigating potentials which are slight variations of the Lennard-Jones poential and which can therefore be seen as providing insights about the behaviour of perturbed Lennard-Jones potentials. Dellago and Posch (1996) investigated two-dimensional particles moving under a potential where the long range part of the Lennard-Jones potential has been replaced by a cubic spine. They found that the motion appears to be epsilon-ergodic and solutions seem to diverge exponentially on average (the largest Lyapunov exponent was positive) for the energy levels corresponding to gases (all two-particles interactions were considered). Donnay (1999) considers two-dimensional generalized Lennard-Jones potentials, a broad class of smooth potentials that are attracting for large $r$ and approach infinity at some point as $r$ goes to zero

\footnotetext{
${ }^{12}$ With respect to correct relaxation times, Bocchieri et al. (1970), Mountain and Thirumalai (1989) and Yoshimura's (1997) results indicate that Lennard-Jones gases approach equilibrium very quickly, namely, in less than $10^{-3}$ seconds.

${ }^{13}$ The phase space volume of the regular region is large and not negligible.

${ }^{14}$ As acknowledged in the papers listed in this paragraph, another possibility is that the motion below the energy threshold is actually epsilon-ergodic but does not appear so because the approach to equilibrium is extremely slow.

${ }^{15}$ This is also one of the main reasons why the Markus-Meyer theorem is no threat to our claim that small perturbations in the Whitney topology of Lennard-Jones Hamiltonians are typically epsilon-ergodic for the energy values relevant to gases. All the Markus-Meyer theorem shows is that epsilon-ergodicity breaks down for very low energy values - but these values do not correspond to gases (see Frigg and Werndl 2011a).
} 
and where, additionally, there is a cutoff radius outside which the potential is zero. He proves that the movement of two particles under such potentials are not ergodic for some values of the energy. However, he is quick to remark that for a higher number of particles the system is more likely to be ergodic. Furthermore, Donnay (1999) remarks that even if generalized Lennard-Jones potentials with a higher number of particles should turn out not to be ergodic, they are likely to be epsilon-ergodic: "Even if one could find such examples [generalized Lennard-Jones systems with a large number of particles that are nonergodic], the measure of the set of solutions constrained to lie near the elliptic periodic orbits is likely to be very small. Thus from a practical point of view, these systems may appear to be ergodic." Finally, Stoddard and Ford (1973) investigated a two-dimensional gas where the Lennard-Jones potential was slightly modified by introducing a cutoff radius outside which the potential is zero (all two-particles interactions were considered). They found evidence for epsilon-ergodicity and for exponential divergence of nearby solutions (for the system being a C-system - see Arnold and Avez 1968).

While these numerical studies do not add up to a strict proof, they provide good reasons to believe that Lennard-Jones potentials and their purturbations are epsilon-ergodic.

\section{Completing the Argument}

The argument in the last section shows that the more restricted claim as an interpretation of the typicality claim involved in Premise 2 is strongly supported by numerical evidence. We submit that the evidence is in fact strong enough to accept this claim. The above typicality argument can be stated more precisely as follows:

Premise 1: The macrostate structure of the gas is such that equilibrium states are m-typical in $\Gamma_{E}$.

Premise 2: The Hamiltonian of the gas is t-typical in $L$.

Conclusion: Typical initial conditions lie on solutions exhibiting TD-like behaviour.

It now remains to be shown that the conclusion indeed follows from the premises. By now this is relatively straightforward. By definition a Hamiltonian is t-typical if it is epsilon-ergodic. In such systems the set $B$ of initial 
conditions that do not lie on non-ergodic solutions are at most of measure $\varepsilon$. Even if all these conditions lie in $\Gamma_{M_{p}}$, we have $\mu_{E}(B) / \mu_{E}\left(\Gamma_{M_{p}}\right) \leq \varepsilon / \mu_{E}\left(\Gamma_{M_{p}}\right)$. Now set $\varepsilon / \mu_{E}\left(\Gamma_{M_{p}}\right):=\delta$. Since $\varepsilon$ is very small by assumption, $\varepsilon / \mu_{E}\left(\Gamma_{M_{p}}\right)$ will be small too and initial conditions lying on non-ergodic solutions are m-atypical in $\Gamma_{M_{p}}$; hence initial conditions lying on ergodic solutions are mtypical. By Premise 1 the largest part of $\Gamma_{E}$ is taken up by equilibrium states $M_{e q}$ and therefore an ergodic solution behaves TD-like. Since ergodic solutions are m-typical in $\Gamma_{M_{p}}$ with respect to $\mu_{E}(\cdot) / \mu_{E}\left(\Gamma_{M_{p}}\right)$, TD-like solutions are m-typical in $\Gamma_{M_{p}}$ with respect to $\mu_{E}(\cdot) / \mu_{E}\left(\Gamma_{M_{p}}\right)$.

The open question is whether this argument could also be run with $G$ rather than $L$, and if such a widening of the scope is desirable at all. As we have mentioned above, it is entirely unclear how to specify $G$. In the absence of such general arguments one may well want to rethink one's wish list. Is it even desirable to proof a more general version of Premise 2? The answer may well be 'no'. As a matter of fact many relevant gases are well described by Lennard-Jones potentials and so for these systems it is enough to drive the point home for this potential. If another class of systems requires another potential function, the way forward would be to prove the equivalent of the typicality claim involved in Premise 2 for that potential function. So the suggestion is that it is sufficient to prove typicality claims for those potentials that are empirically relevant, rather than for an entire class of functions many of which may not have any physical relevance for the specific system under consideration. This more piecemeal approach has the advantage that it seems empirically more relevant because it gives us what we need for the specific system under consideration, and it avoids unnecessary complications about specifying $G$.

\section{References}

Arnold, Vladimir I. and André Avez 1968. Ergodic Problems of Classical Mechanics. New York: W.A. Benjamin.

Attard, Phil 2002. Thermodynamics and Statistical Mechanics: Equilibrium by Entropy Maximisation. London and San Diego: Academic Press.

Bennetin, Giancarlo and Alexander Tenenbaum 1983. "Ordered and Stochastic Behavior in a Two-Dimensional Lennard-Jones System". Physics Review A 28:3020-29. 
Bocchieri, P., Antonio Scotti, Bruno Bearzi and A. Loinger 1970. "Anharmonic Chain With Lennard-Jones Interaction." Physical Review A 2:213-9.

Boltzmann, Ludwig 1877. "Über die Beziehung zwischen dem zweiten Hauptsatze der mechanischen Wärmetheorie und der Wahrscheinlichkeitsrechnung respektive den Sätzen über das Wärmegleichgewicht". Wiener Berichte 53:373-435.

Dellago, Christop and Harald A. 1996. "Lyapunov Instability, Local Curvature, and the Fluid-solid Phase Transition in Two-dimensional Particle Systems". Physical Review A 230:364-387.

Donnay, Victor J. 1999. "Non-Ergodicity of Two Particles Interacting Via a Smooth Potential." Journal of Statistical Physics 96:1021-48.

Frigg, Roman 2009. "Typicality and the Approach to Equilibrium in Boltzmannian Statistical Mechanics." Philosophy of Science (Proceedings) 76:997-1008.

2010a. "Probability in Boltzmannian Statistical Mechanics". In Time, Chance and Reduction. Philosophical Aspects of Statistical Mechanics, eds. Gerhard Ernst and Andreas Hüttemann, 92-118. Cambridge: Cambridge University Press.

2010b. "Why Typicality Does Not Explain the Approach to Equilibrium." In Probabilities, Causes and Propensitites in Physics, ed. Mauricio Suárez, 77-93. Berlin: Springer.

Frigg, Roman, and Carl Hoefer 2010. "Determinism and Chance From a Humean Perspective." In The Present Situation in the Philosophy of Science, ed. Dennis Dieks, Wesley Gonzalez, Stephan Harmann, Marcel Weber, Friedrich Stadler, and Thomas Uebel, 351-72. Berlin and New York: Springer.

Frigg, Roman and Werndl, Charlotte. 2011a. "Explaining Thermodynamiclike Behaviour in Terms of Epsilon-ergodicity". Philosophy of Science 78:628-52.

2011b. "Entropy A Guide for the Perplexed." In Probability in Physics, ed. Claus Beisbart and Stephan Hartmann, 115-42. Oxford: Oxford University Press.

Goldstein, Sheldon 2001. "Boltzmann's Approach to Statistical Mechanics." In Chance in Physics: Foundations and Perspectives, ed. Jean Bric- 
mont, Detlef Dürr, Maria C. Galavotti, Gian C. Ghirardi, Francesco Pettrucione, and Nino Zanghi, 39-54. Berlin and New York: Springer.

Goldstein, Sheldon and Joel L. Lebowtiz 2004. "On the (Boltzmann) Entropy of Nonequilibrium Systems", Physica D 193:53-66.

Hansen, Jean-Pierre and Loup Verlet 1969. "Phase Transitions of the LennardJones System". Physical Review 184:151-61.

Hirsch, Morris W. 1976. Differential Topology. Berlin: Springer.

Lavis, David 2005. "Boltzmann and Gibbs: An Attempted Reconciliation." Studies in History and Philosophy of Modern Physics 36:245-73.

Lebowitz, Joel L. 1993a. "Boltzmanns Entropy and Time's Arrow". Physics Today (September):32-8.

Lebowitz, Joel L. 1993b. "Macroscopic Laws, Microscopic Dynamics, Time's Arrow and Boltzmann's Entropy". Physica A 194:1-27.

Lebowitz, Joel L. and Oliver Penrose 1979. "Modern Ergodic Theory", Physics Today February:1-7.

Markus, Larry and Kenneth R. Meyer 1969. "Generic Hamiltonians Are Not Ergodic." In Proceedings of the 9th Conference on Nonlinear oscillations, ed. Yu A. Mitropolsky, 311-32. Kiev: Kiev Naukova Dumka.

1974. "Generic Hamiltonian Dynamical Systems Are Neither Integrable Nor Ergodic." Memoirs of the American Mathematical Society 144:1-52.

McQuarrie, Donald A. 2000. Statistical Mechanics. Sausolito, California: University Science Books.

Mountain, Raymund D. and D. Thurimalai 1989. "Measures of Effective Ergodic Convergence in Liquids'. Journal of Physical Chemistry 93:697579.

Oxtoby, John C. 1980. Measure and Category. New York, Heidelberg, Berlin: Springer.

Reichl, Linda E. 1998. A Modern Course in Statistical Physics. New York: Wiley.

Robinson, Clark 1995. Dynamical Systems. Stability, Symbol Dynamics and Chaos, Tokyo: CRC Press.

Saxena, E. 1957. "Thermal Conductivity of Binary and Ternary Mixtures of Helium, Argon and Xenon". Indian Journal of Physics 31:597-606. 
Stoddard, Spotswood D., and Joseph Ford. 1973. "Numerical Experiments on the Stochastic Behaviour of a Lennard-Jones Gas System." Physical Review A 8:1504-12.

Thornton, E. 1960. "Viscosity and Thermal Conductivity of Binary Gas Mixtures: Xenon-Krypton, Xenon-Argon, Xenon-Neon and Xenon-Helium", Proceedings of the Physical Society 76:104-12.

Verlet, Loup 1967. "Computer "Experiments" on Classical Fluids. I. Thermodynamical Properties of Lennard-Jones Molecules". Physical Review 159: 98-103.

Vranas, Peter B.M. 1998. "Epsilon-Ergodicity and the Success of Equilibrium Statistical Mechanics." Philosophy of Science 65:688-708.

_ 2009c. "What Are the New Implications of Chaos for Unpredictability?" The British Journal for the Philosophy of Science 60:195-220.

Yoshimura, Kazuyoshi 1997. "Strong Stochasticity Threshold in Some Anharmonic Lattices." Physica D 104:148-62.

Zabaloy, Marcelo S., Vasquez, Victor R. Eugénia A. Macedo 2006. "Description of Self-diffusion Coefficients of Gases, Liquids and Fluids at High Pressure Based on Molecular Simulation Data". Fluid Phase Equilibria 242:43-56. 